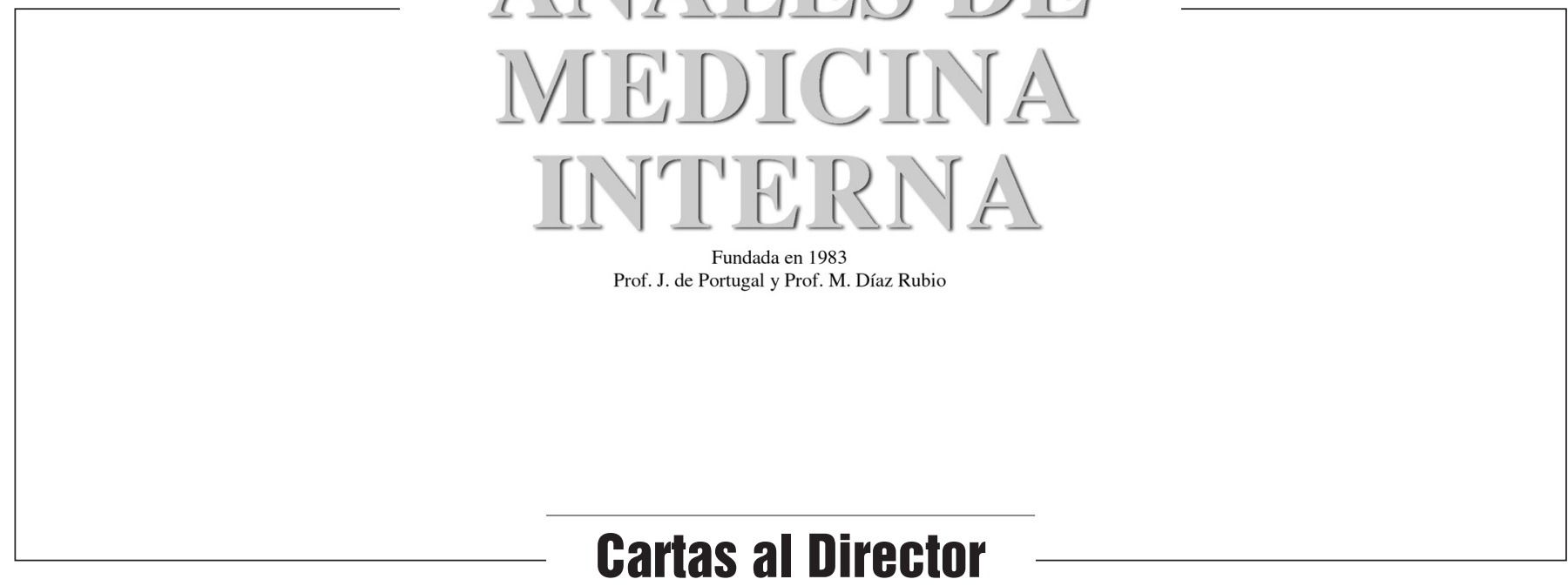

\section{Enfermedad por reflujo gastroesofágico: características de los pacientes derivados desde Atención Primaria}

\section{Sr. Director:}

La enfermedad por reflujo gastroesofágico (ERGE) es uno de los motivos de consulta más frecuentes en atención primaria, y por extensión en las de los especialistas en patología digestiva en las consultas externas del medio hospitalario.

En estudios realizados sobre la población española en 2004 se ha establecido una prevalencia del 31,6\% (1). Se estima que en nuestro medio entre el 4-7\% de la población presenta pirosis diaria y hasta un $30-44 \%$ pirosis mensual. En la población adulta, el 25-65\% de las personas padecen síntomas compatibles con reflujo gastroesofágico en algún momento de sus vidas, con una demanda de asistencia médica en un $28 \%$ de ellos.

La alta prevalencia y el elevado consumo de recursos sanitarios que conlleva su tratamiento han animado a las distintas sociedades científicas a crear guías de práctica clínica con el fin de orientar un manejo óptimo del problema. En este sentido, nace en la "Guía de práctica clínica sobre ERGE" (2) elaborada conjuntamente por la sociedad española de medicina de familia y comunitaria y por la asociación española de gastroenterología, y validada por el centro Cochrane Iberoamericano en el seno del programa de "elaboración de guías clínicas en enfermedades digestivas desde atención primaria a especializada" desarrollada por dichas sociedades.

Esta guía se basa en la mejor evidencia disponible, y su objetivo es orientar a los profesionales en el manejo adecuado de la ERGE y fomentar una utilización adecuada de los recursos sanitarios disponibles. Pretende facilitar la coordinación entre niveles asistenciales, médicos especialistas en atención primaria y médicos especialistas en aparato digestivo, homogeneizando la actuación entre los diferentes profesionales.

El éxito de este tipo de documentos se basa en una adecuada difusión e implantación entre los profesionales. Nuestro objetivo es el de conocer las características clínicas y demográficas de nuestros pacientes derivados desde atención primaria por ERGE y conocer las pautas de actuación que previamente se había realizado sobre ellos, utilizando como validación los algoritmos propuestos en la "Guía de práctica clínica sobre ERGE”, por ser un instrumento de reconocido prestigio y validado por las sociedades científicas de los especialistas implicados.

Hemos realizado el seguimiento en consulta externa de un total de 274 pacientes derivados como primera visita a lo largo de dos meses. Se seleccionaron 87 pacientes cuyo motivo de derivación ha sido la ERGE en cualquiera de sus expresiones clínicas y se les sometió a un cuestionario que permitió conocer sus características clínicas y demográficas, así como las actuaciones realizados sobre ellos desde los centros de salud. Los pacientes que acudían derivados desde otras áreas que no fueran atención primaria o que venían para revisión de patologías previamente estudiadas en nuestras consultas no fueron incluidos en el estudio. Tampoco se incluyeron pacientes derivados por otras patologías que no fueran propiamente la ERGE o en los que no quedara suficientemente claro el motivo de derivación. Para evitar sesgos de selección, no se advirtió a los médicos de atención primaria sobre la realización del estudio, evitando así que su actuación se viera condicionada y permitiéndonos conocer la situación real de la implantación de la guía de práctica clínica. Tampoco se realizó previamente ningún curso de formación ni programa de actualización en ERGE.

Se ha establecido que del total de consultas generadas desde atención primaria para valoración en consulta externa de digestivo, el 35,3\% de las mismas tenia como motivo la valoración de pacientes con ERGE. El 55\% de los pacientes incluidos en el estudio eran varones y un $45 \%$ mujeres con una media de edad de 43,2 $\pm 3,4$ años de edad. Un $36,4 \%$ se reconocía como fumador activo y un $8,5 \%$ consumía alcohol de forma habitual.

El 70,5\% llevaba más de un año de evolución y el 14,1\% más de seis meses antes de ser valorado en nuestra consulta. El 51,7\% presentaba síntomas diariamente y el 38,4\% al menos dos o tres veces en semana. La pirosis, definida como sensación de quemazón o ardor que surge del estómago o bajo tórax y que asciende hacia le cuello, estaba presente en el 90,5\% de casos, y la regurgitación ácida, definida como el retorno sin esfuerzo del contenido gástrico al esófago o la boca, en un $84,7 \%$. En el 58,8\% la clínica tenía un predominio posprandial y en un $51,1 \%$ el paciente había sufrido algún episodio previo de despertar nocturno en relación con clínica de ERGE.

Preguntados sobre la actuación que se había realizado sobre ellos en el centro de salud, el 58,8\% de los pacientes reconoce que el médico el ha explicado en que consiste la enfermedad y como modificar los hábitos y estilos de vida, aunque solo el $48,2 \%$ ha seguido estos consejos. Un $84,7 \%$ ha recibido un trata- 
miento farmacológico indicado por su médico de cabecera, de los cuales, un $71,7 \%$ fue un inhibidor de la bomba de protones (el IBP usado con mas frecuencia es Omeprazol, 63,3\%), un 20\% recibió tratamiento con un antiácido, siendo el más frecuente el Almagato, un 17,6\% utilizó un procinético, siendo el más usado la Cinitaprida, y en un $3,5 \%$ un antiH2, donde el más utilizado fue la Ranitidina.

Entre los motivos de derivación, el 53,5\% lo hizo por respuesta insuficiente a tratamiento empírico, un $15,4 \%$ por presencia de síntomas de alarma, el 8\% para valoración en el diagnostico diferencial de síntomas atípicos. Se ha establecido tras analizar los datos, que en un 78,7\% los criterios de derivación a consulta externa de aparato digestivo se adecua a los algoritmos establecidos en la "Guía de práctica clínica sobre ERGE".

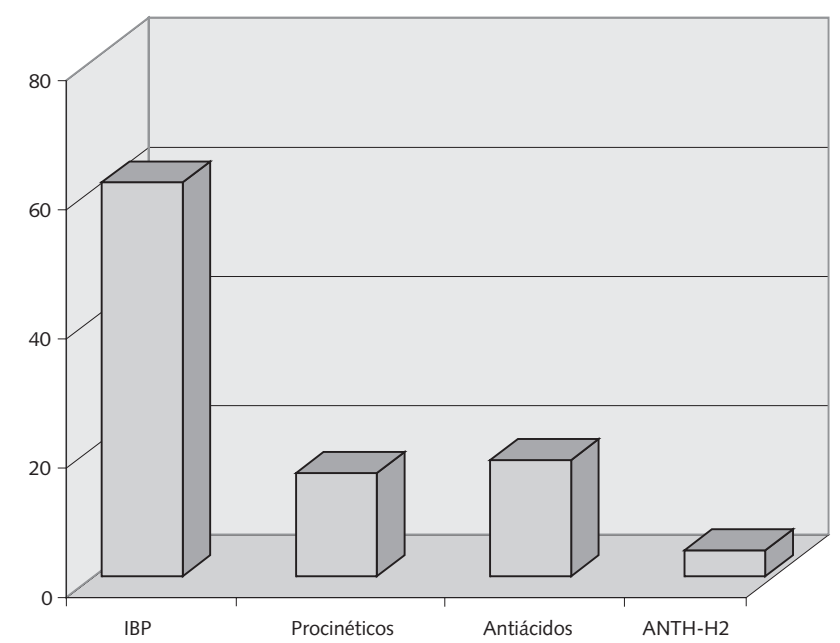

Fig. 1. Tipos de tratamientos utilizados previamente a la valoración en consulta externa.

Pese a considerarse una enfermedad benigna, la persistencia e intensidad de los síntomas puede interferir con las actividades de la vida cotidiana y producir una considerable morbilidad Esto es lo que genera un gran número de consultas, que hace que en España un $28 \%$ de los pacientes con ERGE hayan consultado con su médico en el último año (3). En nuestro medio el 35,3\% del total de las primeras visitas derivadas desde atención primaria se relacionaba con la ERGE, lo que implica una importante carga de trabajo en consultas externas.

Estudios previos realizados en atención primaria han analizado la concordancia entre síntomas de pirosis y regurgitación y la endoscopia (prueba que no se considera como patrón de referencia) y sus resultados muestran que los médicos de atención primaria son capaces de diagnosticar la ERGE a partir de los síntomas de manera bastante precisa (4). Además, cabe recordar que no existe una buena correlación entre la intensidad o frecuencia de los síntomas y la gravedad de las lesiones endoscópicas (5).

Respecto al tipo de tratamiento realizado previamente a la derivación hacia el medio hospitalario, se considera que el ensayo terapéutico con inhibidores de las bombas de protones (IBP) es útil para el diagnóstico de la ERGE y es una prueba más simple y mejor tolerada que la endoscopia y la monitorización del $\mathrm{pH}$ (6). En nuestro caso, el algoritmo diagnostico de la "Guía de práctica clínica sobre ERGE" recomienda dicho ensayo previo a evaluar otras medidas. Nuestro grupo de pacientes había realizado tratamiento previamente en un $84,7 \%$, de los cuales el $71,7 \%$ fueron con IBP, por lo general usados a dosis estándar durante 4 semanas.

La atención a los pacientes con ERGE no obliga a un esfuerzo importante en utilización de recursos sanitarios ya que en nuestra serie supone el 35,3\% del total de pacientes derivados como primera visita desde atención primaria. Las guías de actuación clínica permitan una coordinación entre niveles y una actuación coordinada entre médicos de familia y atención hospitalaria. En este sentido es necesario destacar el alto nivel de derivación que cumple los criterios propuestos.

El 78,7\% de los pacientes estaban derivados correctamente, lo que supera nuestras expectativas iniciales y habla muy favorablemente del trabajo realizado por los médicos de atención primaria. Destacamos también que hasta un 58,8\% de los pacientes consideraba que conocía cuales eran las características de su enfermedad y que hábitos de vida debía modificar.

\section{J. I. Ramos-Clemente Romero, M. A. Pérez Ramos, A. Tala- vera Fabuel $^{1}$, H. Pallarés Manrrique ${ }^{1}$, A. Maraver García ${ }^{1}$}

Servicio de Medicina Interna. ${ }^{1}$ Unidad de Patología Digestiva. Hospital Juan Ramón Jiménez. Huelva

1. Díaz-Rubio M, Moreno Eloa-Olaso C, Rey E, Locke GR, Rodríguez Artadejo F. Symptoms of Gastro-oesophageal Reflux: prevalence, severity, duration and associated factors in a Spanish population. Aliment Pharmacol Ther 2004; 9: 95-105.

2. Marzo M, Alonso P, Bonfill X, Fernández M, Ferrandiz J, Martínez G, et al. Guía de práctica clínica sobre el manejo del paciente con enfermedad por reflujo gastroesofágico (ERGE) Gastroenterol Hepatol 2002; 25: 85-110.

3. Rey E, Moreno Eloas-Olaso C, Rodríguez Artadejo F, Díaz-Rubio M. Medical consultation for gastro-oesophageal reflux symptoms: reasons and associated factors. Digestión 2004; 70: 173-177.

4. Enck P, Dubois D, Marquis P. Quality of life in patients with upper gastrointestinal symptoms: results from the Domestic/International Gastroenterology Surveillance Study (DIGEST). Scand J Gastroenterol 1999; 231(Supl.): 48-54.

5. Dent J, Brun J, Fendrick AM, Fennerty MB, Janssens J, Kahrilas PJ, et al. An evidence-based appraisal of reflux disease management-The Genval Workshop Report. Gut 1999; 44 (Supl. 2): S1-S16.

6. Schindlbeck NE, Klauser AG, Voderholzer WA, Müller-Lissner SA. Empiric therapy for gastroesophageal reflux disease. Arch Intern Med 1995; 155: 1808-12.

\section{La consulta de Medicina Interna puede funcionar en un alto porcentaje de casos como una consulta de alta resolución}

\section{Sr. Director:}

La consulta externa es el área asistencial de mayor importancia en las especialidades médicas. Debe ser el lugar idóneo donde diagnosticar y establecer la estrategia terapéutica de los pacientes enviados desde atención primaria. Por otra parte, la hospitalización debe ser únicamente un instrumento de apoyo, utilizable cuando fuera estrictamente necesario y durante el menor tiempo posible (1). 